\title{
Mycobacterium agri Skin Infection in a Previously Healthy Patient: A Case Study
}

\author{
Chengan $\mathrm{Xu}\left(\mathbb{D}^{1,2}\right.$ \\ Wenhao $\mathrm{Wu}^{2}$ \\ Hongyi $\operatorname{Pan}^{3}$ \\ Tianchen $\mathrm{Hui}^{2}$ \\ Qingqing $\mathrm{Wu}^{2}$ \\ Zhewen Zhou ${ }^{2}$ \\ Shouhao Wang ${ }^{2}$ \\ Wei Zheng ${ }^{2}$ \\ Qiaoqiao $\mathrm{Yi}^{2}$ \\ Hongying $\operatorname{Pan}^{2}$ \\ 'The Second Clinical Medical College, \\ Zhejiang Chinese Medical University, \\ Hangzhou, Zhejiang, 310053, People's \\ Republic of China; ${ }^{2}$ Department of \\ Infectious Diseases, Zhejiang Provincial \\ People's Hospital, Affiliated People's \\ Hospital, Hangzhou Medical College, \\ Hangzhou, Zhejiang, 3I00I4, People's \\ Republic of China; ${ }^{3}$ Department of \\ Internal Medicine, Pujiang County \\ People's Hospital, Pujiang Branch of the \\ first affiliated Hospital of Zhejiang \\ University, Jinhua, Zhejiang, 322200, \\ People's Republic of China
}

Correspondence: Hongying Pan

$\mathrm{Tel} / \mathrm{Fax}+86-57 \mathrm{I}-8589-3603$

Email hypanzjsrmyy@126.com

\begin{abstract}
Nontuberculous mycobacteria infections present mostly pulmonary characteristics. However, the incidence of skin and soft tissue infections caused by nontuberculous mycobacteria has increased in part due to the increased popularity of cosmetic and plastic surgery. Here, we report a case of Mycobacterium agri infection. The patient underwent a one-year course of anti-infection therapy. To the best of our knowledge, this is the first report of a previously healthy patient presenting a skin and soft tissue infection caused by Mycobacterium agri. Clinical personnel should be aware of possible causes of persistent skin and soft tissue infection after cosmetic and plastic surgery.
\end{abstract}

Keywords: Mycobacterium agri, nontuberculous mycobacteria, skin and soft tissue infection, cosmetic and plastic surgery

\section{Introduction}

Nontuberculous mycobacteria (NTM) infections present mostly pulmonary manifestations. However, skin and soft tissue infections are common with rapid growers. ${ }^{1}$ Here, we report the case of a previously healthy patient, who developed an infection with Mycobacterium agri (a rapidly growing nontuberculous bacillus) that was isolated from purulent samples. Treatment of NTM is challenging due to multiple antimycobacterial drug resistances. However, in vitro sensitivity is not always correlated to in vivo sensitivity. ${ }^{2}$ Consideration of drug sensitivity tests and antimicrobial therapy combinations is important to improve patient outcomes.

\section{Case}

A 45-year-old woman without significant past medical history presented to the Plastic Surgery Department of our hospital on June 26, 2020 for evaluation of erythematous nodules, swelling, and pain in her left upper arm that had developed 40 days before.

The patient had received subcutaneous injections of phosphatidylcholine into her left upper arm at a beauty salon on May 15, 2020 (the treatment was meant to promote fat dissolution and achieve local fat reduction). Erythema and pain appeared at the injection sites on May 16, 2020 (Figure 1A). She was treated with antibiotics empirically at the local clinic (oral amoxicillin and intravenous cephalosporins to fight the infection). One week later, the patient complained of large purulent discharge (Figure 1B), and she was administered intravenous penicillin. After that treatment, the erythema did not resolve and abscesses appeared (Figure 1C). The patient came to the outpatient clinic of our hospital on June 26, 


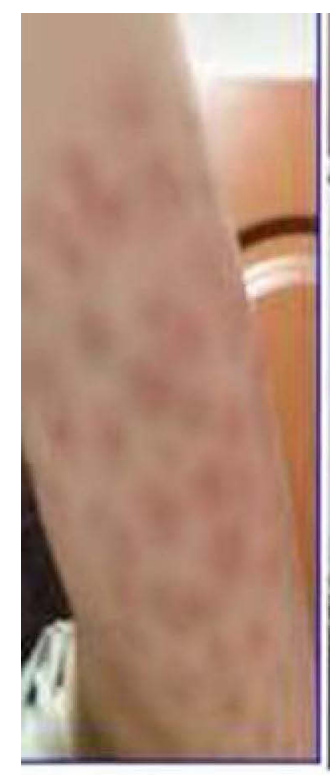

A

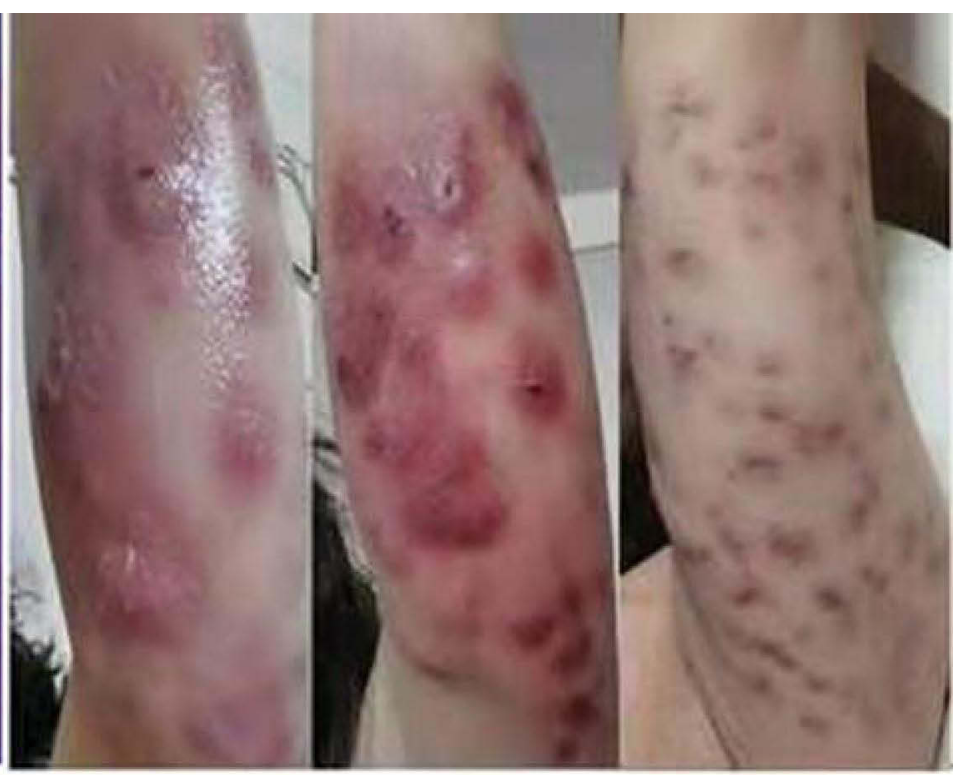

B
C
D

Figure I Evolution of skin in this patient. (A). Erythema and pain at the puncture site one day after the phosphatidylcholine solution administered at a beauty salon. (B) Lesions with purulent discharge. (C) Abscesses. (D) After one-year course of treatment, in May 202I, Resolution of the abscesses after one-year course of treatment with erythema still present.

2020 wihtout systemic symptoms of infection, such as fever, weight loss, respiratory, or gastrointestinal manifestations. In particular, she had no significant lymphadenopathies. Physical examination showed scattered erythematous nodules in the left upper arm, and rupture with purulent fluid was evident. The patient complained of nodule pressure pain.

At our hospital, the arm nodules were punctured, and we took pus samples for bacterial cultures. Acid-fast bacilli (AFB) cultures were negative, but $M$. agri grew on medium and was identified by the MALDI-TOF MS system after five days, which confirmed the infection with NTM. The broth microdilution MIC determination for susceptibility testing following CLSI guidelines (M100, 2020) showed

Table I Antimicrobial Susceptibility Tests for Mycobacterium agri

\begin{tabular}{|l|l|l|}
\hline Antibiotics & MICS $(\boldsymbol{\mu g} / \mathbf{m L})$ & Susceptibility \\
\hline Amikacin & 8 & Susceptible \\
Ciprofloxacin & 32 & Resistant \\
Clarithromycin & 32 & Resistant \\
Imipenem & 32 & Resistant \\
Linezolid & 256 & Resistant \\
Rifampicin & 6 & Resistant \\
\hline
\end{tabular}

susceptibility for amikacin, and resistance against ciprofloxacin, imipenem, linezolid, and rifampicin (Table 1).

Based on the susceptibility testing results, we initiated antibiotic therapy with intramuscular amikacin (400 mg twice a day). The symptoms did not improve after ten days of treatment. The patient was admitted to our hospital on July 07, 2020. Laboratory examinations showed 6.40 $\mathrm{x} 10^{\wedge} \mathrm{9} / \mathrm{L}$ leukocytes and $2.1 \mathrm{mg} / \mathrm{L}$ of C-reactive protein in serum, the erythrocyte sedimentation rate and procalcitonin level were normal. Human immunodeficiency virus and hepatitis B virus serology tests were negative. Liver and renal function tests, and a chest X-ray showed results within normal limits.

The patient was started on treatment with parenteral amikacin (400 mg once a day), oral ethambutol (1000 mg once a day) and clarithromycin (500 mg twice a day). In vitro drug susceptibility testing was not performed. Based on our experience with other NTM skin infections, in vitro sensitivity results rarely correlate with in vivo sensitivity. ${ }^{3}$ No specific treatment recommendations for $M$. agri exist, so we customized the therapy on the basis of the most frequently encountered species. To our knowledge, some cases respond well to clarithromycin combined with other antimicrobial drugs. ${ }^{3-5}$ Our patient had a good response to the combination antibiotic therapy after two days, with 
resolution of the erythematous nodules, swelling, and pain. On July 11, 2020, the erythema of the arm was re-punctured.

Bacterial and fungal cultures, as well as acid-fast evaluation were all negative. The patient was discharged three days later. Persistent infected cutaneous lesions were noted on follow-up outpatient appointments. The treatment was changed to an oral regimen consisting of oral ethambutol (1000 mg once a day) combined with oral clarithromycin (500 mg twice a day). Five months later, the erythema had not decreased (exudation was not present), and some pain remained; thus, we changed the antibiotic therapy to oral doxycycline (100 mg once a day), oral clarithromycin (500 mg once a day) and oral compound sulfamethoxazole (800 mg twice a day) on December 8, 2020. Continuous clinical improvement was noted. In May 2021, the localized pain had disappeared, but the erythema persisted (Figure 1D).

\section{Discussion}

Cosmetic invasive operations for aesthetic reasons have become popular. Complications (such as infections) have increased proportionally to the total number of procedures. Reports of skin infections caused by NTM have increased in numbers. 1,6

NTM grow in soil and water throughout the world. Based on the growth rate of mycobacteria on solid culture media, most NTM belong to the rapidly growing mycobacteria (RGM). ${ }^{3}$ These include ${ }^{3}$ clinically relevant species (M. fortuitum, M. abscessus, and M. chelonae) that are causing an increasing number of skin and soft tissue infections, which generally manifest with symptoms of cellulitis, erythematous nodules, abscesses, subcutaneous nodules (pseudoerythema nodosum), and ulcerations. ${ }^{2}$

Multiple reports have shown outbreaks of $M$. abscessus infections after Mohs surgery, liposuction, injection, soft tissue augmentation, and acupuncture. ${ }^{7-9}$ In addition, infections caused by $M$. fortuitum, ${ }^{10}$ and more rarely with $M$. chelonae, ${ }^{11}$ have also been reported.

Clinically, RGM infections affect predominantly immunocompromised patients, ${ }^{12,13}$ and they are highly resistant to common first-line anti-tuberculosis drugs requiring a combination of multiple drugs and an extended treatment course. ${ }^{14}$ Published treatment recommendations and guidelines are mostly based on the clinical experience of experts and on anti-microbial susceptibility test results. ${ }^{15} M$. agri, belongs to the rapidly growing $\mathrm{NTM}^{16}$ and colonies on growth media plates are visible within 7 days; the bacillus is found ubiquitously in soil and water systems. No evidence for animal-to-human or human-tohuman transmission exists, and infections in humans are infrequent. Skin and soft-tissue infections cause local discomfort quickly, but systemic symptoms are rare. The infection does not respond well to antibiotics and persists for a long time. In our case, the patient had no other preexisting medical conditions, and she developed local symptoms after the first cosmetic injection, her skin manifestations were misdiagnosed as an infection caused by Staphylococcus aureus or Streptococcus pyogenes (more common bacterial pathogens).

We sent the purulent fluid samples from the patient's nodules for bacterial and fungal cultures. We had planned to culture phosphatidylcholine solution or syringe swab samples from the beauty salon, but they refused to cooperate. Given the single point of the infection initiation at the puncture site, we assumed the phosphatidylcholine solution, the operating room equipment, or the patient's own skin were harboring the bacterium before the inoculation.

The variety of NTM causing human infections is increasing. Physicians should suspect an NTM etiology in patients with persistent skin and soft tissue infections without systemic symptoms, who fail to respond to conventional therapy after cosmetic and plastic surgery procedures, or after subcutaneous fatty tissue injections of pharmaceutical products, plant extracts, vitamins, or other compounds. ${ }^{17}$ Early skin biopsy and cultures of tissue specimens or material obtained from draining lesions are particularly important for diagnosis. The ${ }^{16}$ $\mathrm{S}$ rRNA gene sequencing standard for defining new species works well. RGM should be identified at the species level using a recognized acceptable methodology, such as PRA or biochemical testing. In vitro antimicrobial susceptibility testing should be carried out using a broth microdilution protocol. ${ }^{18}$ The bacterium isolated from our patient was shown to be sensitive to amikacin (one of the first-line drugs for NTM), ${ }^{19}$ and resistant to a number of antibiotics. However, considering that in vitro sensitivity rarely correlates to in vivo sensitivity, we prescribed an amikacin therapy including other antibiotics. In conclusion, the lack of established therapies or therapeutic studies based on large groups of patients means patients often require months of combined antimicrobial therapy (chosen according to susceptibility testing and the experience of treating clinicians) before resolution is seen. ${ }^{3}$

NTM infections have been linked to individuals performing cosmetic procedures in their homes or under sub- 
sterile conditions. To prevent these infections, the public should be well informed to avoid the risk of alternative medicine practices that provide injections of unapproved substances. Similarly, operators in beauty salons should always use clean, sterilized instruments and use an aseptic technique to prevent contaminations. After the procedure, the surgical wound and injection site should not be exposed to tap water or tap water-derived liquids to prevent infections with NTM.

\section{Abbreviation}

NTM, nontuberculous mycobacteria.

\section{Ethics Approval and Consent for Publication}

This study has been reviewed and approved to publish the case details by the Research. Ethics Committee of the Zhejiang Provincial People's Hospital University. The patient provided consent for publication of the clinical details, and written informed consent was obtained.

\section{Acknowledgments}

Chengan $\mathrm{Xu}$ and Hongying Pan are co-first authors for this study.

\section{Disclosure}

The authors declare that they have no conflicts of interest.

\section{References}

1. Lin DJ, Wong TT, Ciavarra GA, et al. Adventures and misadventures in plastic surgery and soft-tissue implants. Radiographics. 2017;37 (7):2145-2163. doi: $10.1148 / \mathrm{rg} .2017170090$

2. Forbes BA, Hall GS, Miller MB, et al. Practice guidelines for clinical microbiology laboratories: mycobacteria. Clin Microbiol Rev. 2018;31 (2):e00038-17. doi:10.1128/CMR.00038-17.

3. Griffith DE, Aksamit T, Brown-Elliott BA, et al. An official ATS/ IDSA statement: diagnosis, treatment, and prevention of nontuberculous mycobacterial diseases. Am J Respir Crit Care Med. 2007;175 (4):367-416. doi:10.1164/rccm.200604-571ST

4. Veraldi S, Pontini P, Nazzaro G. Amputation of a finger in a patient with multidrug-resistant Mycobacterium marinum skin infection. Infect Drug Resist. 2018;11:2069-2071. doi:10.2147/IDR.S179815

Infection and Drug Resistance

\section{Publish your work in this journal}

Infection and Drug Resistance is an international, peer-reviewed openaccess journal that focuses on the optimal treatment of infection (bacterial, fungal and viral) and the development and institution of preventive strategies to minimize the development and spread of resistance. The journal is specifically concerned with the epidemiology of
5. Rallis E, Koumantaki-Mathioudaki E. Treatment of Mycobacterium marinum cutaneous infections. Expert Opin Pharmacother. 2007;8 (17):2965-2978. doi:10.1517/14656566.8.17.2965

6. Schnabel D, Gaines J, Nguyen DB, et al. Notes from the field: rapidly growing nontuberculous Mycobacterium wound infections among medical tourists undergoing cosmetic surgeries in the Dominican Republic-multiple states, March 2013-February 2014. MMWR Morb Mortal Wkly Rep. 2014;63(9):201-202.

7. Fisher EJ, Gloster HM. Infection with mycobacterium abscessus after Mohs micrographic surgery in an immunocompetent patient. Dermatol Surg. 2005;31(7):790-794. doi:10.1097/00042728200507000-00014

8. Ryu HJ, Kim WJ, Oh CH, et al. Iatrogenic Mycobacterium abscessus infection associated with acupuncture: clinical manifestations and its treatment. Int J Dermatol. 2005;44(10):846-850. doi:10.1111/j.13654632.2005.02241.x

9. Toy BR, Frank PJ. Outbreak of Mycobacterium abscessus infection after soft tissue augmentation. Dermatol Surg. 2003;29(9):971-973.

10. Seo DH, Shin JY, Roh SG, et al. Non-tuberculous Mycobacterium infection after transfer of autologous fat to the face: a rare case. $\mathrm{Br}$ $J$ Oral Maxillofac Surg. 2019;57(2):185-187. doi:10.1016/j. bjoms.2018.12.008

11. Knox KR, Granick MS, Mitchell AT, et al. Infection with nontuberculous mycobacterium after injection of adulterated silicone fluid. Aesthet Surg J. 2004;24(4):342-345. doi:10.1016/j.asj.2004.04.003

12. Piersimoni C, Scarparo C. Extrapulmonary infections associated with nontuberculous mycobacteria in immunocompetent persons. Emerg Infect Dis. 2009;15(9):1351-1358; quiz 1544. doi:10.3201/ eid1509.081259

13. Gonzalez-Santiago TM, Drage LA. Nontuberculous mycobacteria: skin and soft tissue infections. Dermatol Clin. 2015;33(3):563-577. doi:10.1016/j.det.2015.03.017

14. Johansen MD, Herrmann JL, Kremer L. Non-tuberculous mycobacteria and the rise of Mycobacterium abscessus. Nat Rev Microbiol. 2020;18(7):392-407. doi:10.1038/s41579-020-0331-1

15. Wu ML, Aziz DB, Dartois V, et al. NTM drug discovery: status, gaps and the way forward. Drug Discov Today. 2018;23(8):1502-1519. doi:10.1016/j.drudis.2018.04.001

16. Tsukamura M, Ichiyama S. Numerical classification of rapidly growing nonphotochromogenic mycobacteria. Microbiol Immunol. 1986;30(9):863-882. doi:10.1111/j.1348-0421.1986.tb03014.x

17. Green DA, Whittier S, Greendyke W, et al. Outbreak of rapidly growing nontuberculous Mycobacteria among patients undergoing cosmetic surgery in the Dominican Republic. Ann Plast Surg. 2017;78(1):17-21. doi:10.1097/SAP.0000000000000746

18. Franco-Paredes C, Marcos LA, Henao-Martínez AF, et al. Cutaneous mycobacterial infections. Clin Microbiol Rev. 2018;32(1):e0069-18. doi:10.1128/CMR.00069-18.

19. Brown-Elliott BA, Woods GL, Kraft CS. Antimycobacterial susceptibility testing of nontuberculous mycobacteria. J Clin Microbiol. 2019;57(10):e00834-19. doi:10.1128/JCM.00834-19

\section{Dovepress}

antibiotic resistance and the mechanisms of resistance development and diffusion in both hospitals and the community. The manuscript management system is completely online and includes a very quick and fair peerreview system, which is all easy to use. Visit http://www.dovepress.com/ testimonials.php to read real quotes from published authors. 